\title{
Erratum to: Percutaneous insertion of Zilver stent in malignant biliary obstruction
}

Y. H. Han, M.-Y. Kim, S. Y. Kim, Y. H. Kim, Y. J. Hwang, J. W. Seo, S. J. Cha, G. Hur

Department of Radiology, Ilsan Paik Hospital, Medical College of Inje University, 2240, Daewha-dong, Ilsan-gu, Goyang-si, Gyonggi-do 411-706, Korea

Erratum to: Abdom Imaging (2006) 31:433-438 DOI 10.1007/s00261-005-8017-8

This article was inadvertently published a second time under DOI: 10.1007/s00261-006-9103-2.

Official publication is under DOI: 10.1007/s00261-005-8017-8 appearing in Abdom Imaging (2006) 31:433-438.

The online version of the original article can be found under doi:10.1007/s00261-005-8017-8.

Correspondence to: Y. H. Han; email: hanyoonhee@ilsanpaik.ac.kr 